This item was submitted to Loughborough's Research Repository by the author.

Items in Figshare are protected by copyright, with all rights reserved, unless otherwise indicated.

\title{
Advanced Ginzburg-Landau theory of freezing: a density-functional approach
}

PLEASE CITE THE PUBLISHED VERSION

https://doi.org/10.1103/PhysRevB.90.104101

\section{PUBLISHER}

(C) American Physical Society

\section{VERSION}

AM (Accepted Manuscript)

\section{PUBLISHER STATEMENT}

This work is made available according to the conditions of the Creative Commons Attribution-NonCommercialNoDerivatives 4.0 International (CC BY-NC-ND 4.0) licence. Full details of this licence are available at: https://creativecommons.org/licenses/by-nc-nd/4.0/

\section{LICENCE}

CC BY-NC-ND 4.0

\section{REPOSITORY RECORD}

Toth, Gyula I., and Nikolas Provatas. 2019. "Advanced Ginzburg-landau Theory of Freezing: A Densityfunctional Approach". figshare. https://hdl.handle.net/2134/27304. 


\title{
Advanced Ginzburg-Landau theory of freezing I: A density functional approach
}

\author{
Gyula I. Tóth \\ Institute for Solid State Physics and Optics, \\ Wigner Research Centre for Physics, \\ P.O. Box 49, H-1525 Budapest, Hungary and \\ Institute of Physics and Technology, University of Bergen, \\ Allégaten 55, N-5007 Bergen, Norway* \\ Nikolas Provatas \\ Department of Physics and Centre for the Physics of Materials, \\ McGill University, 3600 Rue University, Montreal, Canada H3A-2T8
}

(Dated: December 6, 2014)

\begin{abstract}
This paper re-visits the weakly fourth order anisotropic Ginzburg-Landau (GL) theory of freezing (also known as Landau-Brazowskii model or theory of weak crystallization) by comparing it to the recent density functional approach of the the Phase-Field Crystal (PFC) model. First we study the critical behavior of a generalized PFC model and show that (i) the so-called one-mode approximation for the Phase-Field Crystal model is exact, and (ii) the direct correlation function has no contribution to the phase diagram in the leading order. Next, we calculate the anisotropy of the crystal-liquid interfacial free energy in the Phase-Field Crystal (PFC) model analytically. For comparison, we also determine the anisotropy numerically and show that no range of parameters can be found for which the Phase-Field Crystal equation can quantitatively model anisotropy for metallic materials. Finally, we derive the leading order PFC amplitude model and show that it coincides with the weakly fourth order anisotropic GL theory, as a consequence of that the assumptions of the GL theory are inherent in the PFC model. We also propose a way to calibrate the anisotropy in the Ginzburg-Landau theory via a generalized gradient operator emerging from the direct correlation function appearing in the generating PFC free energy functional.
\end{abstract}

\section{INTRODUCTION}

The anisotropy of the crystal-liquid interfacial free energy is regarded as the key factor of dendritic solidification, since it determines the microstructure of the crystallizing material, including many commercial metallic alloys. Many attempts have been made to determine the shape and the value of the anisotropy of the interfacial free energy, including equilibrium shape measurements [1-3] and molecular dynamics simulations. Molecular dynamics-based methods, such as the cleaving technique $[4-7]$ and the capillary fluctuation method $[8,9]$ predict the anisotropy in the order of $1 \%$ for several metallic systems. (For bcc systems, see References [10, 11].) Since it has been revealed that the anisotropy critically depends on the crystal symmetry, and its magnitude depends mostly on the ratio of the crystal-liquid interface thickness and the interatomic distance, continuum descriptions also can be relevant tools for describing the anisotropic properties.

The first order parameter theory that captures anisotropy was developed by Haymet and Oxtoby $[12,13]$. The description is based on the classical Density Functional Theory (DFT) of freezing of the Ramakrishnan-Yussouff type [14], which characterizes the system by the time-averaged local one-particle den-

\footnotetext{
* Gyula.Toth@ift.uib.no
}

sity. Since the theory works on the molecular scale in space, it inherently contains the crystalline symmetries of the system. Later a more convenient description, the Ginzburg-Landau (GL) theory of bcc-liquid interfaces was developed by Shih et al [15]. (The theory is also known as weak crystallization theory or LandauBrazowskii theory [16].) In the GL theory the free energy of nonuniform phases is expressed in terms of spacedependent reciprocal lattice vector amplitudes, which are constant in the bulk phases and vary on the scale of the crystal-liquid interface thickness. The original theory of Shih et al [15] and its revised version by Wu et al [17] predicts the anisotropy parameter for iron of $\nu=0.0222$ and 0.0237 , respectively, where the anisotropy parameter is defined as $\nu:=\left(\gamma_{100}-\gamma_{111}\right) /\left(\gamma_{100}+\gamma_{111}\right)$, and where $\gamma_{100}$ and $\gamma_{111}$ are the interfacial free energies for the [100] and [111] crystal-liquid equilibrium planar interfaces, respectively. A recent, DFT motivated approach, the PhaseField Crystal (PFC) model [18] predicts $\nu=0.026[19]$ and $\nu=0.03$ [20], while the PFC amplitude model [19] similarly yielded $\nu=0.03$.

Although the results of continuum theories are fair agreement with the experimental results and the results of atomistic simulations, both the $4^{\text {th }}$-order GL and PFC amplitude theories of pure materials have a quite worrisome common property pointed out by Majaniemi and Provatas [21]: since all material parameters (except the crystal structure) scale out from the free energy functional, the anisotropy parameter in these models de- 
pends exclusively on the crystal structure but not on the temperature, for instance, which limits the applicability of these theories, and necessitates further development of GL models. The starting point of developing advanced anisotropic Ginzburg-Landau theories is the classical Density Functional Theory (DFT), since the classical DFT inherently contains the crystal symmetries. The Phase-field Crystal model is a simplified $4^{\text {th }}$-order classical density functional theory which is relatively easy to handle analytically, and, moreover, its amplitude theory is quite similar to the anisotropic GL theory [19], which seems to be more than just a coincidence.

The paper is organized as follows: In Section II we discuss the invariant formulations of the Phase-Field Crystal free energy functional and find the most convenient form (the so-called "minimal form") to work with. In Section III we calculate the equilibrium properties of the bulk (liquid and crystal) phases, and determine the critical properties (critical exponents and coefficients) of the equilibrium crystal amplitude and equilibrium density in the gapless approximation (i.e. when the crystal-liquid equilibrium density gap is neglected). Using the results, we prove that the one-mode approximation is exact in the leading order. In Section IV we calculate the interfacial free energy in the PFC analytically and numerically, and show that because of the presence of a remnant anisotropy the PFC control parameter cannot be used to quantify the anisotropy in metallic materials. In Section $\mathrm{V}$ we show that weakly fourth order Ginzburg-Landau theory is apparently the leading order amplitude theory of the PFC. Finally, we propose a method to calibrate the anisotropy parameter in the GL via a generalized gradient operator emerging from the direct correlation function in the PFC. In section VI we discuss and compare our results to the results of previous works.

\section{THE PHASE-FIELD CRYSTAL MODEL}

In the first part we investigate the crystal-liquid equilibrium in the Phase-Field Crystal model introduced in Ref. [18]. After defining the free energy functional, we investigate the behavior of the PFC model close to the critical point, and prove that the first reciprocal lattice vector (RLV) set dominance of the model is related to the critical exponents of the RLV set amplitudes.

In the single-component Phase-Field Crystal model the free energy of the system relative to a reference homogeneous state of density $\rho_{0}$ reads as $[20,22]$ :

$$
\frac{\Delta F}{\rho_{0} k_{B} T}=\int d \mathbf{r}\left\{n \frac{1-\rho_{0} \hat{C}_{2}}{2} n-a \frac{n^{3}}{3}+b \frac{n^{4}}{4}\right\},
$$

where $n(\mathbf{r})=\left[\rho(\mathbf{r})-\rho_{0}\right] / \rho_{0}$ is the scaled density field, and $C_{2}(k)$ is a single-peaked direct correlation function in the wavelength space with peak position $k_{0}$ (see Fig 1.a). As a first step, we scale the model in order to identify the important parameters: Scaling the length as $\mathbf{r}=\lambda \cdot \tilde{\mathbf{r}}$,
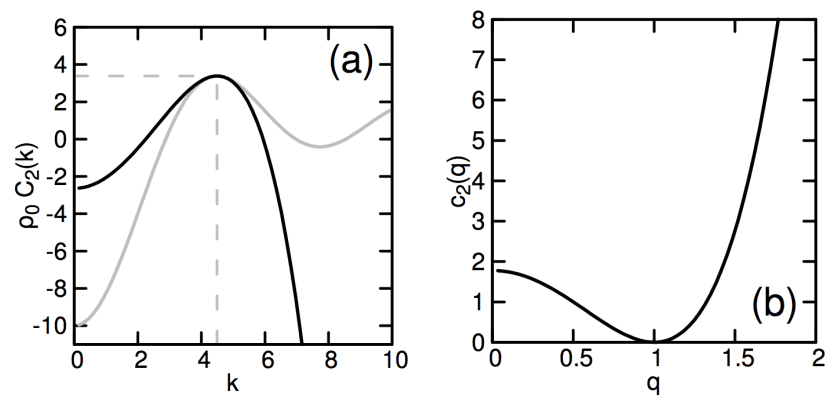

FIG. 1. Direct correlation functions: (a) Schematic correlation function of a real system (gray) and typical Phase-Field Crystal correlation function (black). (b) Scaled PFC correlation function $c_{2}(q)=1-C_{2}\left(q \cdot k_{0}\right) / C_{2}\left(k_{0}\right)$, where $k_{0}$ is the position of the maximum of the PFC $C_{2}(k)$ (indicated by the horizontal dashed gray line in panel a). Note that the zerovalued minimum of $c_{2}(q)$ at $q=1$ is independent from the particular form of the PFC $C_{2}(k)$.

the order parameter as $n=X \cdot \phi$ and the free energy as $\Delta F /\left(\rho_{0} k_{B} T\right)=A \cdot \tilde{F}$ results in a simplified form of Eq. (1):

$$
\tilde{F}=\int d \tilde{\mathbf{r}}\left\{\phi \frac{\hat{c}_{2}-r}{2} \phi-t \frac{\phi^{3}}{3}+\frac{\phi^{4}}{4}\right\}
$$

The choice of $\lambda:=1 / k_{0}$ and $c_{2}(q):=\left[C_{2}\left(k_{0}\right)-C_{2}(q\right.$. $\left.\left.k_{0}\right)\right] / v$ results in the scales $X=\sqrt{\rho_{0} v / b}$ and $A=$ $\left[\rho_{0} v\right]^{2} /\left(k_{0}^{3} b\right)$, and the parameters $r=\left[\rho_{0} C_{2}\left(k_{0}\right)-1\right] /\left(\rho_{0} v\right)$ and $t=a / \sqrt{b \rho_{0} v}$, where $v>0$ is an arbitrary scaling parameter. Note that $c_{2}(q)$ is a non-negative function with a single minimum at $q_{0}=1$ with $c_{2}(1)=0$ (see Fig 1.b). This transformation of the direct correlation function will play a crucial role in our derivation: taking into account that $c_{2}(q)$ is an even function, it can be written as $c_{2}(q)=\sum_{i=0}^{\infty} \alpha_{i} q^{2 i}$, which corresponds to $\hat{c}_{2}=\sum_{i=0}^{\infty} \alpha_{i}\left(-\nabla^{2}\right)^{i}$ in real space. (For the sake of simplicity, we won't use . hereafter.) Consequently, the term $\phi \hat{c}_{2} \phi=\sum_{i=0}^{\infty} \alpha_{i}(-1)^{i} \phi\left[\nabla^{2 i} \phi\right]$ in Eq. (2) is equivalent to $\sum_{i=0}^{\infty} \alpha_{i}\left(\nabla^{i} \phi\right)^{2}$ in the variational sense (i.e. both formulae results in the same functional derivative with respect to $\phi)$. Using this equivalence, the cubic term $-t\left(\phi^{3} / 3\right)$ can be eliminated by substituting $\phi=\psi+t / 3$ into Eq. (2). It simply results in $\phi \hat{c}_{2} \phi \rightarrow \psi \hat{c}_{2} \psi$, while the terms up to the first order in $\psi$ can be neglected (since such terms vanish in both the Euler-Lagrange equation and the equation of motion). The "minimal" form of the original free energy functional then reads as

$$
F=\int d \mathbf{r}\left\{\psi \frac{\hat{c}_{2}-\epsilon}{2} \psi+\frac{\psi^{4}}{4}\right\}
$$

where $\epsilon=r-t^{2} / 3$. This is a fairly simple form compared to Eq. (1) and shows that the important parameters of the model are only $\epsilon$ and $c_{2}(q)$. 


\section{EQUILIBRIUM PROPERTIES}

In this section first we will investigate the periodic solutions of the PFC functionals, writing up the exact free energy density of a lattice periodic solution. Next, we will set up the equilibrium conditions defining the lattice constant of the crystal lattice, the bulk crystal amplitudes and the equilibrium density in the gapless approximation. In Section C, The critical behavior of the system will be studied in equilibrium, and, considering the critical exponents for the RLV set amplitudes, we will prove that the one-mode approximation (i.e. when only the first reciprocal lattice vector set is considered in the free energy density) is actually exact in the leading order.

\section{A. Periodic solutions}

Eq. (3) generates a first order phase transition between homogeneous (liquid) and lattice periodic (crystal) solutions. These phases represent extrema of the free energy functional, therefore, they can be found by solving the Euler-Lagrange equation (ELE): $\delta F / \delta \psi=\mu$ by definition, where $\delta F / \delta \psi$ is the functional derivative of $F$ with respect to $\psi$, and $\mu=(\delta F / \delta \psi)_{\psi_{L}}$, i.e. the chemical potential of a homogeneous background liquid of density $\rho_{L}$. Since the ELE is a nonlinear, higher order PDE, usually it is solved numerically. Alternatively, a lattice periodic solution is defined by:

$$
\psi_{p}(\mathbf{r})=\bar{\psi}+\sum_{I} A_{I} \sum_{i \in S(I)} \exp ^{\imath \Gamma_{i}^{I} \cdot \mathbf{r}}
$$

where $\bar{\psi}$ is the average density, $A_{I}$ the amplitude of the $I^{t h}$ RLV set, and $\Gamma_{i}^{I}$ the $i^{t h}$ RLV in the $I^{t h}$ RLV set. The bulk free energy density is defined as the volumetric average of the free energy in a unit cell:

$$
f\left[\psi_{p}\right]:=\frac{1}{V_{\text {cell }}} \int_{V_{\text {cell }}} d V\left\{I\left[\psi_{p}\right]\right\},
$$

where $I[$.$] is the integrand of Eq. (3). For practical$ reasons we define the free energy density difference as $\Delta f\left[\psi_{p}\right]:=f\left[\psi_{p}\right]-f[\bar{\psi}]$. Using this together with Eqns. (4) and (3), and considering $\psi \cdot \hat{c}_{2}[\psi]=\sum_{i=0}^{\infty} \alpha_{i}\left(\nabla^{i} \psi\right)^{2}$ results in the exact free energy density of a lattice periodic solution (see Appendix A):

$$
\begin{aligned}
\Delta f\left[\psi_{p}\right]= & \sum_{I}\left[A_{I}^{2} N_{I, I}^{(2)}\right] \frac{c_{2}\left(\Gamma_{I}\right)-\epsilon+3 \bar{\psi}^{2}}{2}+ \\
& +\bar{\psi} \sum_{I, J, K}\left(A_{I} A_{J} A_{K}\right) \mathcal{N}_{I, J, K}^{(3)} \\
& +\frac{1}{4} \sum_{I, J, K, L}\left(A_{I} A_{J} A_{K} A_{L}\right) \mathcal{N}_{I, J, K, L}^{(4)},
\end{aligned}
$$

where we introduced the shorthand notation

$$
\mathcal{N}_{I_{1}, I_{2}, \ldots, I_{N}}^{(N)}:=\sum_{i_{1}, i_{2}, \ldots, i_{N}} \delta_{i_{1}, i_{2}, \ldots, i_{N}}^{I_{1}, I_{2}, \ldots, I_{N}}
$$

where $\delta_{i_{1}, i_{2}, \ldots, i_{N}}^{I_{1}, I_{2}, \ldots, I_{N}}$ denoted here as the Kronecker-delta function $\delta\left(\boldsymbol{\Gamma}_{i_{1}}^{I_{1}}+\boldsymbol{\Gamma}_{i_{2}}^{I_{2}}+\cdots+\boldsymbol{\Gamma}_{i_{N}}^{I_{N}}\right)$, which gives 1 if the sum of the reciprocal lattice vectors in the argument is zero, otherwise it is 0 . Therefore, $\mathcal{N}_{I_{1}, I_{2}, \ldots, I_{N}}^{(N)}$ is just the total number of $\mathrm{N}$-term vector sums resulting in zero in which the first vector is from the RLV set $I_{1}$, the second is from $I_{2}$ and so on. Consequently, $N_{I, I}^{(2)}$ is just the number of RLVs in the $I^{t h} \mathrm{RLV}$ set. Note that $\mathcal{N}_{I_{1}, I_{2}, \ldots, I_{N}}^{(N)}$ is invariant for any permutation of the indices.

\section{B. Equilibrium conditions}

Eq. (6) has to be minimized with respect to the set amplitudes $A_{I}$ and the selected wavelength $\Gamma_{I}$ at a constant average density $\bar{\psi}$. Introducing $\Gamma_{I}=\beta_{I} q$, where $\beta_{1}=1$, the minimization equations read as:

$$
\frac{\partial \Delta f\left[\psi_{p}\right]}{\partial A_{I}}=0 \quad \text { and } \quad \frac{\partial \Delta f\left[\psi_{p}\right]}{\partial q}=0
$$

From Eq. (8) two qualitatively different types of solutions emerge: (i) the trivial solution: $A_{I} \equiv 0$ for $I=1 \ldots \infty$ (homogeneous solution, the liquid phase), and (ii) a nontrivial lattice periodic solution (crystalline phase), where $A_{I} \neq 0$. Since in case of $\epsilon \rightarrow 0$ the crystal-liquid equilibrium density jump can be neglected (see Appendix B for details), the equilibrium condition is simply defined by equal free energy densities of the phases at the same average density, i.e.

$$
f[\bar{\psi}]=f\left[\psi_{p}\right] \quad \Rightarrow \quad \Delta f\left[\psi_{p}\right]=0,
$$

where $\psi_{p}$ is the nontrivial solution. Eq. (9) together with Eq. (8) defines the atomic distance $q$, the equilibrium solid amplitudes $A_{I}$ and the equilibrium density $\bar{\psi}$ as a function of $\epsilon$ and $c_{2}(q)$.

\section{Critical behavior}

In this section we show that the general PFC model described by Eq. (1) generates a mean-field Brazowskii/Swift-Hohenberg critical point at $\epsilon=0$. We determine the critical exponents of the equilibrium density $\left(y_{\psi}\right)$ and crystal RLV set amplitudes $\left(y_{I}\right)$ and show that $y_{1}<y_{I}$ for any $I>1$, implying the one-mode dominance of the model.

\section{Wavelength selection}

For the particular choice $c_{2}(q)=\left(1-q^{2}\right)^{2}$ Eq. (3) reduces to the well-known Brazowskii/Swift-Hohenberg form, which has a critical point at $\epsilon=0$ [23]. It is reasonable to assume that this behavior doesn't depend on the particular form of $c_{2}(q)$, and the model has a critical point as long as $c_{2}(q)$ is a positive semidefinite function with a single, zero-value minimum at $k=1$, i.e. 
$c_{2}(1)=0$. Indeed, it is relatively easy to see that the only solution of Eqns. (8) and (9) for $\epsilon=0$ is $\bar{\psi}=0$ and $A_{I}=0$. Therefore, we can write

$$
A_{I}=a_{I} \epsilon^{y_{I}}+\text { h.o.t. and } \bar{\psi}=c_{\psi} \epsilon^{y_{\psi}}+\text { h.o.t. }
$$

for $\epsilon \rightarrow 0$ in general. In order to determine the critical exponents first we assume that there are more than one dominant RLV sets, meaning that $y_{I_{1}}=y_{I_{2}}=\cdots=$ $y_{I_{N}}\left(=: y_{A}\right)$, where $N>1$ and $y_{J}>y_{A}$ for all $J \neq$ $I_{1}, I_{2}, \ldots I_{N}$. Using this, the leading order term of Eq. (9) reads as:

$$
\sum_{I \in\left\{I_{1}, I_{2}, \ldots, I_{N}\right\}} a_{I}^{2} \mathcal{N}_{I, I}^{(2)} c_{2}\left(\beta_{I} q_{0}\right)=0,
$$

where $q_{0}$ is the selected wavelength satisfying $\left.\left(\partial \Delta f\left[\psi_{p}\right] / \partial q_{0}\right)\right|_{q_{0}}=0$. Since $a_{I}^{2} \mathcal{N}_{I, I}^{(2)}>0$ and $c_{2}(q) \geq 0$, Eq. (11) can be satisfied only if $c_{2}\left(\beta_{I} q\right)=0$ for all dominant RLV sets. Since $c_{2}(q)$ has only one minimum at $q_{0}=1$ for which $c_{2}(1)=0$, only one RLV set can be dominant. In addition, this must be the first RLV set (thus $q=q_{0}$ ), since we're searching for a crystal structure (in other words, the only dominant RLV set cannot be a higher harmonic). Moreover, since $c_{2}(1)=0$, the term $\psi \hat{c}_{2} \psi$ has no effect on the phase diagram. This is in accordance with the original assumption, that the existence of the critical point doesn't depend on the particular choice of $c_{2}(q)$. The critical point exists as long as $c_{2}(q) \geq 0$ and has a single minimum at $q_{0}=1$ with $c_{2}\left(q_{0}\right)=0$.

\section{Critical exponents}

Taking into account that $y_{I}>y_{A}$ for $I>1$ and using $q_{0}=1$, the equilibrium condition reads as:

$$
\begin{aligned}
& \Delta f\left[\psi_{p}\right]=A_{1}^{2} N_{1} \frac{3 \bar{\psi}^{2}-\epsilon}{2}+\bar{\psi} A_{1}^{3} N_{3}+\frac{A_{1}^{4}}{4} N_{4}+ \\
& +\sum_{I>1}\left[A_{I}^{2} N_{I} c_{2}\left(\beta_{I}\right)+\right. \\
& \left.+3 \bar{\psi} A_{1}^{2} A_{I} \mathcal{N}_{1,1, I}^{(3)}+A_{1}^{3} A_{I} \mathcal{N}_{1,1,1, I}^{(4)}+\text { h.o.t. }\right]=0,
\end{aligned}
$$

where we used the shorthand notations $N_{I}:=\mathcal{N}_{I, I}^{(2)}$, $N_{3}:=\mathcal{N}_{1,1,1}^{(3)}$ and $N_{4}:=\mathcal{N}_{1,1,1,1}^{(4)}$ (details are shown in Appendix A). From Eq. (4) it is trivial that $y_{A}=y_{\psi}$, otherwise, there is no first order transition for $\epsilon \rightarrow 0$. In addition, in order to find nontrivial solution for $a_{1}$ and $c_{\psi}$, the $\propto \psi^{4}$ term in the free energy functional must contribute to the leading order. Taking these facts into account, the first row of Eq. (12) together with $\partial \Delta f\left[\psi_{p}\right] / \partial A_{I}=0$ implies

$$
y_{A}=y_{\psi}=1 / 2,
$$

therefore, the leading order of Eq. (6) is $\epsilon^{2}$. In the next order of Eq. (12) (the second and the third lines) the minimization equations for $A_{I>1}$ are decoupled: $\frac{\partial \Delta f\left[\psi_{p}\right]}{\partial A_{I}}=$
$2 A_{I} N_{I} c_{2}\left(\beta_{I}\right)+3 A_{1}^{2} \bar{\psi} \mathcal{N}_{1,1, I}^{(3)}+4 A_{1}^{3} \mathcal{N}_{1,1,1, I}^{(4)}+$ h.o.t. $=0$, resulting in

$$
y_{I>1}=3 / 2
$$

on the same basis, therefore, the next order of Eq. (12) is proportional to $\epsilon^{3}$. In addition, from $\partial \Delta f\left[\psi_{p}\right] / \partial q=0$ it can be shown that $q^{2}=1+O\left(\epsilon^{2}\right)$, therefore, the first correction from this in Eq. (12) is in the order of $\epsilon^{4}$. This means that our calculation is self-consistent, therefore, the single-mode approximation of the PFC is exact in the leading order.

Finally, one can determine the coefficients $c_{\psi}$ and $a_{1}$ by substituting $q_{0}=1, A_{1}=a_{1} \sqrt{\epsilon}, A_{I>1}=a_{I} \epsilon^{3 / 2}$ and $\bar{\psi}=c_{\psi} \sqrt{\epsilon}$ into Eq. (6) then taking the leading order of Eqns. (8) and (9). The equations then can be solved analytically for $c_{\psi}$ and $a_{1}$ :

$$
\begin{aligned}
& c_{\psi}=-\sqrt{\frac{N_{1} N_{4}}{3 N_{1} N_{4}-2 N_{3}^{2}}}, \\
& a_{1}=\sqrt{\frac{4 N_{1} N_{3}^{2}}{N_{4}\left(3 N_{1} N_{4}-2 N_{3}^{2}\right)}} .
\end{aligned}
$$

\section{INTERFACIAL FREE ENERGY}

The goal of this section is to identify where the anisotropic contribution to the interfacial free energy comes from. Using the result of the previous section, namely, that the one-mode approximation is exact in the leading order, the anisotropic crystal-liquid interfacial free energy can be calculated analytically relatively easily. Considering the isotropic case first, we determine the interface thickness(es) and the interfacial free energy, and their critical exponents. We will show that the nonvanishing behavior of the anisotropy parameter at the critical point comes from the fact, that the first RLV set has an anisotropic contribution to the leading order. In the final part of this section we will calculate the remnant anisotropy for the bcc structure analytically and verify the result by comparing it to the results of numerical solutions of the Euler-Lagrange equation. We also compare our results to the results of previous works.

\section{A. Definition of the anisotropic crystal-liquid interfacial free energy}

When the density jump between the equilibrium crystal and liquid is neglected, the anisotropic interfacial free energy reads as

$$
\gamma(\mathbf{n})=\int_{-\infty}^{\infty} d \xi\left(\frac{1}{A_{\perp}} \int_{\xi} d A_{\perp}\left\{\Delta I\left[\psi_{s l}\right]\right\}\right),
$$

where $\mathbf{n}$ is the normal of the planar crystal-liquid interface, $\xi=\mathbf{n} \cdot \mathbf{r}$ the orthogonal distance from the in- 
terface, while $\left(1 / A_{\perp}\right) \int_{\xi} d A_{\perp}\{\cdot\}$ denotes an average calculated for a plane parallel to the interface at a constant value of $\xi$. The integrand of Eq. (17) reads as $\Delta I\left[\psi_{s l}(\mathbf{r})\right]=I\left[\psi_{s l}(\mathbf{r})\right]-I[\bar{\psi}]$. Here $\psi_{s l}(\mathbf{r})=\bar{\psi}+\Delta \psi_{s l}(\mathbf{r})$ represents the equilibrium crystal-liquid density distribution, where $\Delta \psi_{s l}(\mathbf{r})$ is approximated as

$$
\Delta \psi_{s l}(\mathbf{r})=\sum_{I} A_{I} \sum_{i \in S(I)} \frac{1+g\left(\xi / \Lambda_{i}^{I}\right)}{2} \exp \left(\imath \boldsymbol{\Gamma}_{i}^{I} \cdot \mathbf{r}\right)
$$

where $A_{I}$ 's are the equilibrium crystal amplitudes. The envelope function satisfies $\left.g\left(\xi / \Lambda_{i}^{I}\right)\right|_{\xi \rightarrow \pm \infty} \rightarrow \pm 1$, respectively, where $\Lambda_{i}^{I}$ is the characteristic interface width of the $i^{t h}$ plane wave in the $I^{t h}$ RLV set [21]. Note that far from the interface Eq. (18) recovers the density distribution of the equilibrium bulk phases: $\left.\psi_{s l}(\mathbf{r})\right|_{\xi \rightarrow+\infty} \rightarrow$ $\psi_{p}(\mathbf{r})$ and $\left.\psi_{s l}(\mathbf{r})\right|_{\xi \rightarrow-\infty} \rightarrow \bar{\psi}$. Using Eqns. (18) and (4) in Eq. (70), after a straightforward but lengthy algebra one can come to a reasonably simple parametrized form of the leading order anisotropic crystal-liquid interfacial free energy (for details, see Appendix C):

$$
\begin{aligned}
& \gamma(\mathbf{n})=A_{1}^{2} \sum_{i}\left[\frac{3 \bar{\psi}^{2}-\epsilon}{8}\left\|\left(g_{i}\right)^{2}-1\right\|+\frac{2 \zeta\left(\mathbf{n} \cdot \boldsymbol{\Gamma}_{i}\right)}{3 \Lambda_{i}^{1}}\right]+ \\
& \frac{A_{1}^{3} \bar{\psi}}{8} \sum_{i, j, k}\left[\sum_{m, n}^{i, j, k}\left\|g_{m} g_{n}-1\right\|\right] \delta_{i, j, k}+ \\
& \frac{A_{1}^{4}}{64} \sum_{i, j, k, l}\left[\left\|g_{i} g_{j} g_{k} g_{l}-1\right\|+\sum_{m, n}^{i, j, k, l}\left\|g_{m} g_{n}-1\right\|\right] \delta_{i, j, k, l},
\end{aligned}
$$

where the sums for $(m, n)$ run for all different pairs in $(i, j, k)$ and $(i, j, k, l)$, and we simply left all the ()$^{1},()^{1,1,1}$ and ()$^{1,1,1,1}$ upper indices. The function

$$
\zeta\left(\mathbf{n} \cdot \boldsymbol{\Gamma}_{i}\right)=\zeta_{0}+\zeta_{1}\left(\mathbf{n} \cdot \boldsymbol{\Gamma}_{i}\right)^{2}
$$

is responsible for the anisotropic contribution [here $\zeta_{0}$ and $\zeta_{1}$ are constants emerging from the particular form of $\left.c_{2}(q)\right]$. For example, for the $c_{2}(q)=\left(1-q^{2}\right)^{2}$ theory (Brazowskii/Swift-Hohenberg) $\zeta_{0}=0$ and $\zeta_{1}=1$, therefore, $\zeta\left(\mathbf{n} \cdot \boldsymbol{\Gamma}_{i}^{1}\right)=\left(\mathbf{n} \cdot \boldsymbol{\Gamma}_{i}\right)^{2}$.

\section{B. Critical exponent of the interface thickness}

Close to the critical point the interface thickness (correlation length) diverge as

$$
\Lambda_{i}^{I}=\lambda_{i}^{I} \cdot \epsilon^{y_{\Lambda}}+\text { h.o.t. },
$$

where $y_{\Lambda}<0$. Note that all interface thicknesses must diverge with a unique critical exponent $y_{\Lambda}$ (for details, see Appendix D.1). In case of the isotropic limit $\left(\Lambda_{i}^{1}=\right.$ $\Lambda_{i}^{2}=\cdots=\Lambda$ ), Eq. (19) reads as:

$$
\gamma_{\text {iso }}=\frac{c_{0}}{\Lambda}+c_{2}\left(3 \bar{\psi}^{2}-\epsilon\right) \Lambda-\left[c_{3} A_{1}^{3}+c_{4} A_{1}^{4}\right] \Lambda
$$

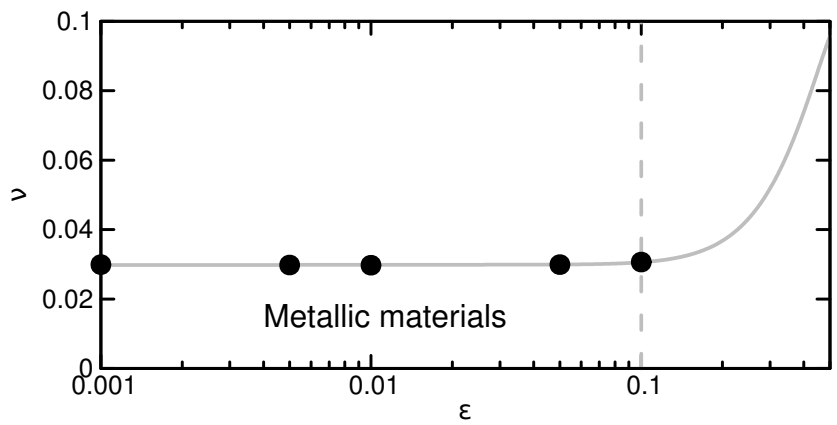

FIG. 2. Crystal-liquid interfacial free energy anisotropy for the $c_{2}(q)=\left(1-q^{2}\right)^{2}$ (Brazowskii/Swift-Hohenberg) model. For $\epsilon<0.1, \nu_{\min }^{\max }=\nu_{111}^{100}$.

where $c_{0}, c_{2}$ and $c_{4}$ are constants. Using $\Lambda=\lambda \cdot \epsilon^{y_{\lambda}}$ (where $\lambda$ is a constant specific to the isotropic case) in the minimization equation $\partial \gamma / \partial \Lambda=0$ yields

$$
y_{\Lambda}=-1 / 2 \text {. }
$$

\section{Critical behavior of the anisotropy}

Using the critical exponents and the fact that $\| g_{i}^{1} g_{j}^{1}-$ $1 \| \propto \epsilon^{y_{\Lambda}}$ and $\left\|g_{i}^{1} g_{j}^{1} g_{k}^{1} g_{l}^{1}-1\right\| \propto \epsilon^{y_{\Lambda}}$ in Eq. (19) yields

$$
\frac{\gamma(\mathbf{n})}{\epsilon^{3 / 2}}=\left(c_{0}^{i}+c_{1}^{i} \epsilon+\ldots\right)+\left[c_{0}^{a}(\mathbf{n})+\epsilon^{2} c_{2}^{a}(\mathbf{n})+\ldots\right]
$$

where the indices ()$^{i, a}$ denote isotropic and anisotropic contributions, respectively. The anisotropy parameter reads as

$$
\nu(\epsilon)=\frac{\left[c_{0}^{a}\left(\mathbf{n}^{+}\right)-c_{0}^{a}\left(\mathbf{n}^{-}\right)\right]+O\left(\epsilon^{2}\right)}{2 c_{0}^{i}+c_{0}^{a}\left(\mathbf{n}^{+}\right)+c_{0}^{a}\left(\mathbf{n}^{-}\right)+O(\epsilon)},
$$

where $\mathbf{n}^{ \pm}$are defined by $\gamma\left(\mathbf{n}^{+}\right):=\max [\gamma(\mathbf{n})]$ and $\gamma\left(\mathbf{n}^{-}\right):=\min [\gamma(\mathbf{n})]$, respectively. From Eq. (19) one can see that $c_{0}^{a}\left(\mathbf{n}^{ \pm}\right) \propto \sum_{i} \zeta\left(\mathbf{n}^{ \pm} \cdot \boldsymbol{\Gamma}_{i}^{1}\right) / \lambda_{i}^{1}$. However, $\lambda_{i}^{1} \neq \lambda_{j}^{1}$ for $i \neq j$ in the case of $\zeta_{1} \neq 0$ in $\zeta(x)$, therefore, the anisotropy is finite at the critical point:

$$
\nu_{\min }^{\max }(\epsilon)=\nu_{0}+O\left(\epsilon^{2}\right) .
$$

This is not surprising as pointed out in Ref [19]: for a vanishing anisotropy the PFC amplitude model should have resulted in $\nu=0$. Note, however, that the remnant anisotropy $\left(\nu_{0}\right)$ is a consequence of the one-mode dominance of the free energy functional, since $y_{1}=1 / 2$ together with $y_{I>1}=3 / 2$ may yield a non-vanishing anisotropic contribution to the leading order of to the interfacial free energy.

\section{Determination of the remnant anisotropy}

To determine $\nu_{0}$ in Eq. (26), first we calculate Eq. (19) divided by Eq. (34) for the general anisotropic case 
using reasonable approximations of the envelope function integrals $\left\|g_{i}^{1} g_{j}^{1}-1\right\|$ and $\left\|g_{i}^{1} g_{j}^{1} g_{k}^{1} g_{l}^{1}-1\right\|$ in Eq. (19) (see Appendix D.2). It yields the coupled minimization equations for interface thickness constants relative to the isotropic one, i.e. $\tilde{\lambda}_{i}=\Lambda_{i}^{1} / \Lambda_{1}$ :

$$
\frac{\partial}{\partial \tilde{\lambda}_{i}}\left[\frac{\gamma(\mathbf{n})}{\gamma_{\text {iso }}}\right]=0 \quad\left(i=1 \ldots N_{1}\right)
$$

which have to be solved numerically for $\tilde{\lambda}_{i}$ for the $c_{2}(q)=$ $\left(1-q^{2}\right)^{2}$ model. For the bcc structure preferred by the Phase-Field Crystal model close to the critical point in 3 dimensions $N_{1}=12, N_{3}=48$ and $N_{4}=540$. We started the numerical calculations from the isotropic solution defined by Eq. (33) for the [111] and [100] crystal planes, which give the minimal and the maximal interfacial free energies, respectively. Our calculation resulted in a significant remnant anisotropy $\nu_{0}=0.026$. It is important to emphasize that this result cannot be regarded exact, since we have used the simplest approximation for $\left\|g_{i}^{1} g_{j}^{1}-1\right\|$ that couples the amplitudes and its simplest extension to $\left\|g_{i}^{1} g_{j}^{1} g_{k}^{1} g_{l}^{1}-1\right\|$. We also have to mention that this approximation doesn't have an effect on our later results, since we won't have to calculate the profile integrals in our derivations anymore.

For comparison to the analytic estimation, following the method of Podmaniczky et al [24], we have evaluated the interfacial free energy by solving the Euler-Lagrange equation $\delta F / \delta \psi=\mu$ numerically for bcc-liquid equilibrium interfaces at $\epsilon=0.001,0.005,0.01,0.05$ and 0.1 . We have found

$$
\nu(\epsilon) \approx 0.03
$$

a nearly constant value, which is in a perfect accordance with the results of Jaatinen et al [20] (numerical solution of the Euler-Lagrange equation) and $\mathrm{Wu}$ and Karma [19] (PFC amplitude model). Nevertheless, we have to mention that in Ref [19] there are two calculations: (i) Numerical simulations with simple diffusive dynamics $\partial_{t} \psi=\nabla^{2}(\delta F / \delta \psi)$ yielded $\nu=0.026$, contrary to the work of Jaatinen, where the Euler-Lagrange equation $\delta F / \delta \psi=\mu$ has been solved. Due to our extensive experience in equilibrium calculations we note that in a simple diffusive dynamics the convergence of the interface is critically slow, practically it never converges to equilibrium unless is was set to the exact solution initially. In contrast, the Euler-Lagrange equation converges at least exponentially, making possible to do such equilibrium calculations. Therefore, in case of the numerical simulations only the result of Jaatinen et al is considered.

At this point we mention that our results can also be relevant for the so-called "anisotropic PFC model" [25]. If there exists such a linear coordinate transformation which diagonalizes the spatial operators in the free energy functional, the problem reduces to the isotropic case studied here, and Eqns. (4) and (18) are valid. This is obviously true for the operator published in Ref. [26].

\section{CONNECTION TO GINZBURG-LANDAU THEORIES}

In this section we will investigate the connection between the leading order amplitude theory of the PhaseField Crystal model and the Ginzburg-Landau theory. Our main objective is, contrary to the results of previous works, to show that the Phase-field Crystal model is identical to the Ginzburg-Landau theory for $\epsilon \rightarrow 0$. In order to prove this, first we derive the isotropic leadingorder amplitude model of the PFC, then - following the recent work of Provatas and Majaniemi - we will extend the derivation for the anisotropic case. Finally we will propose a more general gradient term for the GinzburgLandau theory, and show that the calibration of the interfacial free energy in our generalized Ginzburg-Landau theory is possible via tuning the direct correlation function in the generating density functional theory.

\section{A. Isotropic limit}

Here we first derive the isotropic Ginzburg-Landau polynomial by taking the isotropic limit of the equilibrium PFC free energy density described by Eq. (6), then show that adding a simple gradient square term leads to an isotropic Ginzburg-Landau model, which consistently recovers our results from both the bulk and the interface calculations for the PFC.

\section{Ginzburg-Landau polynomial}

In equilibrium one can define the normalized amplitudes $A_{I}:=\phi_{I}(\mathbf{r}) A_{I}^{0}$, where $\phi_{I}(\mathbf{r}) \in[0,1]$ and $A_{I}^{0}$ denotes the equilibrium amplitudes: $A_{1}^{0}=a_{1} \sqrt{\epsilon}$ and $A_{I>1}^{0}=a_{I} \epsilon^{3 / 2}$. Note that for a planar equilibrium interface $\phi_{I}(x \rightarrow \pm \infty) \rightarrow 0,1$, respectively. With this rescaling, the equilibrium bulk liquid and solid phases are described by $\vec{\phi}_{L}=(0,0,0, \ldots)$ and $\vec{\phi}_{S}=(1,1,1, \ldots)$, respectively. Considering only the leading order terms of Eq. (12) and substituting $A_{1}=A_{1}^{0} \phi$ and $\bar{\psi}=c_{\psi} \sqrt{\epsilon}$ yields

$$
\Delta f(\phi)=w[\phi(1-\phi)]^{2}
$$

where

$$
\frac{w}{\epsilon^{2}}=\frac{4 N_{1}^{2} N_{3}^{4}}{N_{4}\left(3 N_{1} N_{4}-2 N_{3}^{2}\right)^{2}} .
$$

Note that Eq. (29) is exactly the well-known $4^{\text {th }}$ order Ginzburg-Landau polynomial for triangular and bcc structures. (For the fcc structure $N_{3} \equiv 0$, therefore, there is no fcc-liquid first-order phase transition in the SwiftHohenberg formalism in leading order, i.e. close to the critical point.) 


\section{Isotropic gradient operator}

The isotropic single order parameter amplitude equation in equilibrium can be written as:

$$
F_{\text {iso }}^{G L}=\int d V\left\{\kappa(\nabla \phi)^{2}+\Delta f(\phi)\right\},
$$

where $\Delta f(\phi)$ is defined by Eq. (29). The equilibrium solution of the Euler-Lagrange equation $\delta F_{\text {iso }} / \delta \phi=0$ is the kink-function $\phi(x)=[1-\tanh (x / d)] / 2$, where $d=$ $2 \sqrt{\kappa / w}$. The interfacial free energy can be obtained by using the integral Euler-Lagrange equation: $\gamma=\sqrt{\kappa w} / 3$. The model parameters $\kappa$ and $w$ can be then related to the interfacial free energy and interface thickness as:

$$
w=6(\gamma / d) \quad \text { and } \quad \kappa=(3 / 2) \gamma d
$$

The isotropic interface width and interfacial free energy can be determined from the minimization of the isotropic limit of Eq. (19) with respect to $d$ for $g_{i}=$ $[1-\tanh (x / d)] / 2$, yielding

$$
\frac{1}{\epsilon d^{2}}=\left(\frac{1}{8 C}\right) \frac{N_{3}^{2}}{3 N_{1} N_{4}-2 N_{3}^{2}}
$$

and

$$
\frac{\gamma}{\epsilon^{3 / 2}}=\frac{4}{3} \frac{N_{1}^{2}}{N_{4}} \sqrt{2 C}\left(\frac{3 N_{1} N_{4}-2 N_{3}^{2}}{N_{3}^{2}}\right)^{-3 / 2}
$$

where $C=\frac{1}{N_{1}} \sum_{i \in S(1)} \zeta\left(\mathbf{n} \cdot \boldsymbol{\Gamma}_{i}\right)$ is a constant for geometrical reasons. Substituting Eqns. (34) and (33) into Eq. (32) yields

$$
\begin{aligned}
\frac{w}{\epsilon^{2}} & =4\left(\frac{N_{1}^{2}}{N_{4}}\right)\left(\frac{N_{3}^{2}}{3 N_{1} N_{4}-2 N_{3}^{2}}\right)^{2} \\
\frac{\kappa}{\epsilon} & =8 C\left(\frac{N_{1}^{2}}{N_{4}}\right)\left(\frac{N_{3}^{2}}{3 N_{1} N_{4}-2 N_{3}^{2}}\right) .
\end{aligned}
$$

Note that Eq. (35) is identical to Eq. (30), showing that our calculation is self-consistent. Also note that Eq. (33) consistently verifies the divergence of the interface thickness found in Eq. (24).

\section{B. Anisotropic extension}

Using the results of section V.A, we now extend the model to the anisotropic case. As we will see, the anisotropic PFC amplitude model is exactly the Ginzburg-Landau model for the Brazowskii/Swifthohenberg direct correlation function $c_{2}\left(1-q^{2}\right)^{2}$. Besides discussing the results and comparing them to previously published ones, we will propose a generalization of the anisotropic Ginzburg-Landau theory emerging from the general, single-peaked direct correlation function used in the generalized PFC model.

\section{Ginzburg-Landau polynomial}

Introducing the normalized amplitudes $\phi_{i}(\mathbf{r}):=$ $A_{i}^{1} / A_{1}^{0} \in[0,1]$, and substituting $\bar{\psi}=c_{\psi} \sqrt{\epsilon}, A_{1}^{0}=a_{\psi} \sqrt{\epsilon}$ and $c_{2}(1)=0$ into the leading order of Eq. (6) gives:

$$
\begin{aligned}
g(\vec{\phi})= & \frac{\Delta f\left(\left\{\phi_{i}\right\}\right)}{w}=\frac{1}{N_{1}} \sum_{i} \phi_{i}^{2}- \\
& -\frac{2}{N_{3}} \sum_{i, j, k}\left(\phi_{i} \phi_{j} \phi_{k}\right) \delta_{i, j, k}^{1,1,1}+ \\
& +\frac{1}{N_{4}} \sum_{i, j, k, l}\left(\phi_{i} \phi_{j} \phi_{k} \phi_{l}\right) \delta_{i, j, k, l}^{1,1,1,1}
\end{aligned}
$$

where $w$ is defined by Eq. (30). Multiplying Eq. (37) by $a_{2}$ and introducing $\phi_{i}:=u_{i} / u_{s}$ results in the equivalent form

$$
\begin{aligned}
\Delta \tilde{f}(\vec{\phi})= & +a_{2} \sum_{i} c_{i j}\left(u_{i} u_{j}\right) \delta_{i, j}- \\
& -a_{3} \sum_{i, j, k} c_{i j k}\left(u_{i} u_{j} u_{k}\right) \delta_{i, j, k}+ \\
& +a_{4} \sum_{i, j, k, l} c_{i j k l}\left(u_{i} u_{j} u_{k} u_{l}\right) \delta_{i, j, k, l}
\end{aligned}
$$

with $a_{3}=2 a_{2} / u_{s}$ and $a_{4}=a_{2} / u_{s}^{2}$ and $c_{i j}=1 / N_{2}$, $c_{i j k}=1 / N_{3}$ and $c_{i j k l}=1 / N_{4}$. Therefore, Eq. (37) is exactly the anisotropic Ginzburg-Landau theory, since (i) it naturally recovers the ratios $a_{3} / a_{2}$ and $a_{4} / a_{2}$ (this is true by contruction, as mentioned in Ref. [19]), and (ii) inherently contains the Ginzburg-Landau assumption of equal weights $\left(c_{i j}, c_{i j k}\right.$ and $\left.c_{i j k l}\right)$ for the 2, 3 and 4-sided polygons in the RLV space, respectively [15]. Following the methodology proposed by Shih et al [15], for the [110] direction Eq. (37) transforms into Eq. (48) of Ref. [19]. (The calculation can be automated by using advanced symbolic mathematical softwares, such as Mathematica.) On the one hand, it is satisfactory, showing that our calculation is consistent to former works on the PFC amplitude model. On the other hand, it is embarrassing, since Eq. (48) of Ref. [19] and the originally published Ginzburg-Landau model for the [110] direction (Eq. (3.8) in Ref. [15], Eq. (12) in Ref [17] and Eq. (49) in Ref. [19]) differ. In order to find the true reason of this discrepancy, we repeat and extend the argumentation written in Ref. [19]: If the original Ginzburg-Landau polynomial described by Eq. (3.8) in Ref. [15] is correct, the only reason of the difference can be that $c_{i j k l}$ 's differ in the two models. Since in the Ginzburg-Landau model they are equal, they must be different in the PFC amplitude theory, as pointed out in Ref [19]. This immediately leads to a contradiction, since $c_{i j k l}$ 's are equal in Eq. (38), therefore, the original assumption of the argumentation is wrong, namely, the polynomial in Ref. [15] is incorrect. We have to mention that it is quite probable, since (i) our derivation is conistent, (ii) $N_{4}=540$, so there can be formed 540 closed 
4-sided polygons from the 12 bcc first neighbor RLVs, and the original derivation was done in 1987, without using the advantage of present computer technology, and (iii) Refs. [17] and [19] used Eq. (3.8) of [15] only as a reference, without checking its validity.

\section{Generalized gradient operator}

As a final step in deriving the Ginzburg-Landau theory from the PFC, we have to define the anisotropic gradient operator of the GL model. Following Majaniemi and Provatas [21], for a planar interface the anisotropic interfacial free energy can be written as:

$$
\gamma(\mathbf{n})=\int_{-\infty}^{\infty} d \xi\left\{\kappa\left[\left(\partial_{\xi} \vec{\phi}\right)^{T} \cdot \mathbb{S}(\mathbf{n}) \cdot\left(\partial_{\xi} \vec{\phi}\right)\right]+\Delta f[\vec{\phi}(\xi)]\right\},
$$

where $\Delta f[\vec{\phi}]$ and $\kappa$ are defined by Eqns. (37) and (36), respectively. The elements of the coefficient matrix $\mathbb{S}(\mathbf{n})$ can be determined by substituting $\phi_{i}^{*}(\xi)=\left[1+g_{i}(\xi)\right] / 2$ into Eq. (39), and comparing the result with Eq. (19) (for details, see Appendix E). The calculation results in the generalized anisotropic Ginzburg-Landau theory

$$
F_{\mathrm{GL}}=\int d V\left\{\kappa \sum_{i}\left(\nabla \phi_{i}\right)^{T} \cdot \mathbb{A}_{i} \cdot\left(\nabla \phi_{i}\right)+w \cdot g(\vec{\phi})\right\}
$$

where

$$
\mathbb{A}_{i}=\frac{1}{N_{1} C}\left[\zeta_{0} \cdot \mathbb{I}+\zeta_{1} \cdot\left(\boldsymbol{\Gamma}_{i}^{1} \otimes \Gamma_{i}^{1}\right)\right],
$$

while $g(\vec{\phi}), \kappa$ and $w$ are defined by Eqns. (37), (35) and (36), respectively. It is worth to mention that the comparison of Eq. (39) and Eq. (19) was based on only one assumption, namely, that the envelope functions $g_{i}(\xi)$ are identical in the two models (it is not necessary to define the particular shape). Nevertheless, for the Brazowskii/Switf-Hohenberg case, i.e. when $\zeta\left(\mathbf{n} \cdot \Gamma_{i}^{1}\right)=\left(\mathbf{n} \cdot \Gamma_{i}^{1}\right)^{2}$, Eq. (40) coincides with the amplitude model published in Ref [19]. That model yielded $\nu=0.03$ for the anisotropy parameter, exactly the same as our numerical result and the result of Ref. [20] for the GL-PFC model, therefore, our assumption of the identical envelope functions is validated.

It is noteworthy that Eq. (41) defines a general gradient matrix for the Ginzburg-Landau theory. The equivalence of the PFC model and the GL theory opens the possibility of calibrating the anisotropy in the GL theory: $\mathbb{A}_{i}$ depends on the properties of the scaled direct correlation function $c_{2}(q)$ in the generator PFC model via $\zeta_{0}$ and $\zeta_{1}$. In the Brazowskii/Swift-Hohenberg case $\zeta_{0}=0$ and $\zeta_{1}=1$ due to the $4^{\text {th }}$-order direct correlation function (see Appendix C). In general, however, this is not the case: For the eight-order correlation function of
Jaatinen et. al [20], for instance, $\zeta_{0}$ is finite, bringing an isotropic correction to $\zeta_{1}$. Therefore, in this case we expect smaller anisotropy parameter from the GL, which actually has already been calculated in Ref $([20])$, where the corresponding value was $\eta=0.026$. Note that if a closed analytical formula could be set between $\zeta_{0}, \zeta_{1}$ and the polynomial behavior of $c_{2}(q)$, the anisotropy in the GL theory would be easily calibrated. This issue is, however, out of the scope of the present work.

\section{CONCLUSIONS}

Our calculations show that the weakly $4^{\text {th }}$-order anisotropic Ginzburg-Landau theory of freezing is formally analogous to the leading-order amplitude theory of the Brazowskii/Swifth-Hohenberg type Phase-Field Crystal model. The constant anisotropy appearing in weakly $4^{\text {th }}$ order Ginzburg-Landau theories coincides with the critical point remnant anisotropy in the PFC model, and originates from the fact that leading-order anisotropic contribution emerges from the first RLV set in the generating PFC. The equivalence of the PFC model and the GL theory contradicts the results for the [110] direction of previous works, especially as the quartic term in Ref. [15], the value of the anisotropy in Ref. [17] and the argumentation regarding the difference between the GL and the PFC in Ref. [19] for the [110] direction.

Our results have consequences on the quantitative applicability to metals of both the traditional Phase-Field Crystal model and Ginzburg-Landau theories emerging from it. In the case of the PFC model the numerical calculations resulted in a remnant $\left(\nu_{0} \approx 3 \%\right)$ anisotropy

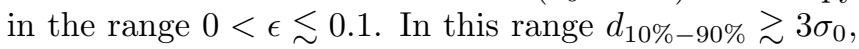
where $d_{10 \%-90 \%}$ is the usual $10 \%-90 \%$ interface thickness and $\sigma_{0}$ the bcc lattice constant. Since this is true for simple metals, $\epsilon$ is not a relevant parameter in quantifying the anisotropy for metallic materials. In contrast, it has been found that $\nu_{0}$ inherited by the GL theory exclusively depends on the form of the scaled direct correlation function $\hat{c}_{2}$. Since the symmetry breaking of the GL coefficient matrix is trivially related to properties of the direct correlation function, one can calibrate the anisotropy in the Ginzburg-Landau theory through the critical behavior of the generating PFC.

A possible pathway of deriving consistent GL theories, in accordance with the original idea of Shih et al [15], is to choose such a PFC description, in which more than one RLV set is dominant, i.e. we at least two peaks of the direct correlation function are considered. A promising candidate is the so-called structural PFC (or XPFC) model [27], in which the peak peak heights are weighted by the Debye-Waller factor. Since the peak heights are not equal, the critical point vanishes, meaning that the $\epsilon$ dependence appears in the amplitude theory. Nevertheless, combining the XPFC model with the recently published fluctuating hydrodynamic theory of freezing 
[28] might result in a continuum description of crystallization of simple liquids on the (classical) fundamental length scale of the material. Moreover, comparing the results of the model with molecular dynamics data will hopefully anchor $\epsilon$ to the physical temperature, making the model fully quantitative.

\section{ACKNOWLEDGEMENTS}

The authors wish to thank professor L. Gránásy and F. Podmaniczky from the Wigner Research Centre for Physics, Hungary, for the valuable discussions which significantly contributed to the quality of the work. This work has been supported by the Postdoctoral Programme of the Hungarian Academy of Sciences and the Natural Sciences and Engineering Research Council of Canada.

\section{APPENDIX A: EVALUATION OF THE BULK FREE ENERGY DENSITY}

In order to evaluate $\Delta f\left[\psi_{p}\right]$ for $\psi_{p}(\mathbf{r})=\bar{\psi}+\Delta \psi(\mathbf{r})$, where $\Delta \psi(\mathbf{r})=\sum_{I} A_{I} \sum_{i \in S(I)} \exp ^{\imath \boldsymbol{\Gamma}_{i}^{I} \cdot \mathbf{r}}$, first we reformulate Eq. (3) as follows:

$$
\mathcal{F}=\int d \mathbf{r}\left\{\frac{1}{2} \sum_{n=0}^{\infty} \alpha_{n}\left(\nabla^{n} \psi\right)^{2}-\epsilon \frac{\psi^{2}}{2}+\frac{\psi^{4}}{4}\right\},
$$

where we used that the functional derivative

$$
\frac{\delta \mathcal{F}}{\delta \psi}=\sum_{i=0}^{\infty}(-1)^{i} \frac{\partial I}{\partial \nabla^{i} \psi}
$$

results in the same for both $\psi \cdot \hat{c}_{2}[\psi]=$ $\sum_{n=0}^{\infty} \alpha_{n} \psi\left[\left(-\nabla^{2}\right)^{n} \psi\right]$ and $\sum_{n=0}^{\infty} \alpha_{n}\left(\nabla^{n} \psi\right)^{2} . \quad$ The spatial derivatives of $\psi(\mathbf{r})$ read as:

$$
\nabla^{n} \psi(\mathbf{r})=\sum_{I} A_{I} \sum_{i \in S(I)}\left({ }^{\Gamma_{i}^{I}}\right)^{n} \exp ^{\imath \boldsymbol{\Gamma}_{i}^{I} \cdot \mathbf{r}},
$$

where $n>1$. Introducing the shorthand notation $\langle\rangle:.=$ $\frac{1}{V_{\text {cell }}} \int_{V_{\text {cell }}} d V\{$.$\} for the lattice cell average the following$ terms emerge from $\psi \cdot \hat{c}_{2}[\psi]$ in the free energy density:

$$
\left\langle\left(\nabla^{n} \psi\right)^{2}\right\rangle=\sum_{I, J} A_{I} A_{J} \sum_{i, j}\left[-\boldsymbol{\Gamma}_{i}^{I} \cdot \boldsymbol{\Gamma}_{j}^{J}\right]^{n}\left\langle\exp ^{\imath\left(\boldsymbol{\Gamma}_{i}^{I}+\boldsymbol{\Gamma}_{j}^{J}\right) \cdot \mathbf{r}}\right\rangle,
$$

where

$$
\left\langle\exp ^{\imath\left(\boldsymbol{\Gamma}_{i}^{I}+\boldsymbol{\Gamma}_{j}^{J}\right) \cdot \mathbf{r}}\right\rangle=\delta\left(\boldsymbol{\Gamma}_{i}^{I}+\boldsymbol{\Gamma}_{j}^{J}\right)
$$

is the (Kronecker) delta-function giving 1 for $\boldsymbol{\Gamma}_{i}^{I}=-\boldsymbol{\Gamma}_{j}^{J}$, and 0 otherwise. Therefore,

$$
\left\langle\left(\nabla^{n} \psi\right)^{2}\right\rangle=\sum_{I} A_{I}^{2} \mathcal{N}_{I, I}^{(2)}\left(\Gamma_{I}\right)^{2 n},
$$

where $\mathcal{N}_{I, I}^{(2)}=\sum_{i, j} \delta\left(\boldsymbol{\Gamma}_{i}^{I}+\boldsymbol{\Gamma}_{j}^{J}\right)$ is just the number of RLVs in the $I^{\text {th }}$ RLV set. Furthermore,

$$
\left\langle\psi^{2}\right\rangle=\bar{\psi}^{2}+\left\langle\Delta \psi^{2}\right\rangle=\bar{\psi}^{2}+\sum_{I} A_{I}^{2} \mathcal{N}_{I, I}^{(2)},
$$

where we used that $\langle\Delta \psi\rangle=0$. Finally,

$$
\frac{1}{2} \sum_{n=0}^{\infty} \alpha_{n}\left\langle\left(\nabla^{n} \psi\right)^{2}\right\rangle=\alpha_{0} \frac{\bar{\psi}^{2}}{2}+\frac{1}{2} \sum_{I} A_{I}^{2} \mathcal{N}_{I, I}^{(2)} \sum_{n=0}^{\infty} \alpha_{n}\left(\Gamma_{I}\right)^{2 n} .
$$

Note that $\sum_{n=0}^{\infty} \alpha_{n}\left(\Gamma_{I}\right)^{2 n} \equiv c_{2}\left(\Gamma_{i}\right)$. Then, the contribution of $\psi \cdot \hat{c}_{2}[\psi]$ to the free energy density reads as:

$$
\frac{1}{2}\left\langle\psi \cdot \hat{c}_{2}[\psi]\right\rangle=\alpha_{0} \frac{\bar{\psi}^{2}}{2}+\frac{1}{2} \sum_{I} A_{I}^{2} \mathcal{N}_{I, I}^{(2)} c_{2}\left(\Gamma_{I}\right) .
$$

Introducing $\mathcal{N}_{I, J, K}^{(3)}:=\sum_{i, j, k} \delta\left(\boldsymbol{\Gamma}_{i}^{I}+\boldsymbol{\Gamma}_{j}^{J}+\boldsymbol{\Gamma}_{k}^{K}\right)$ and $\mathcal{N}_{I, J, K, L}^{(4)}:=\sum_{i, j, k, l} \delta\left(\boldsymbol{\Gamma}_{i}^{I}+\boldsymbol{\Gamma}_{j}^{J}+\boldsymbol{\Gamma}_{k}^{K}+\boldsymbol{\Gamma}_{l}^{L}\right)$, where $i \in$ $S(I), j \in S(J), k \in S(K)$ and $l \in S(L)$, and taking into account that

$$
\left\langle\psi^{4}\right\rangle=\bar{\psi}^{4}+4 \bar{\psi}\left\langle\Delta \psi^{3}\right\rangle+6 \bar{\psi}^{2}\left\langle\Delta \psi^{2}\right\rangle+\left\langle\Delta \psi^{4}\right\rangle,
$$

where

$$
\left\langle\Delta \psi^{3}\right\rangle=\sum_{I, J, K} A_{I} A_{J} A_{K} \mathcal{N}_{I, J, K}^{(3)}
$$

and

$$
\left\langle\Delta \psi^{4}\right\rangle=\sum_{I, J, K, L} A_{I} A_{J} A_{K} A_{L} \mathcal{N}_{I, J, K, L}^{(4)}
$$

yields

$$
\begin{aligned}
f\left[\psi_{p}\right]= & \sum_{I}\left[A_{I}^{2} N_{I, I}^{(2)}\right] \frac{c_{2}\left(\Gamma_{I}\right)-\epsilon+3 \bar{\psi}^{2}}{2}+ \\
& +\bar{\psi} \sum_{I, J, K}\left(A_{I} A_{J} A_{K}\right) \mathcal{N}_{I, J, K}^{(3)} \\
& +\frac{1}{4} \sum_{I, J, K, L}\left(A_{I} A_{J} A_{K} A_{L}\right) \mathcal{N}_{I, J, K, L}^{(4)}+f[\bar{\psi}],
\end{aligned}
$$

where $f[\bar{\psi}]=\left(\alpha_{0}-\epsilon\right)\left(\bar{\psi}^{2} / 2\right)+\bar{\psi}^{4} / 4$. Therefore, $\Delta f\left[\psi_{p}\right]=$ $f\left[\psi_{p}\right]-f[\bar{\psi}]$ results in Eq. (6).

\section{APPENDIX B: INCLUDING THE EQUILIBRIUM DENSITY JUMP}

If one includes the equilibrium crystal-liquid density jump, the relevant thermodynamic potential is grand potential density, which reads as:

$$
\begin{aligned}
\omega_{s} & :=f\left[\psi_{p}\right]-\mu_{s} \psi_{s} \\
\omega_{l} & :=f\left[\psi_{l}\right]-\mu_{l} \psi_{l}
\end{aligned}
$$


where $\psi_{p}(\mathbf{r})=\psi_{s}+\Delta \psi(\mathbf{r})$ is the bulk solid solution, where $\Delta \psi(\mathbf{r})=\sum_{I} A_{I} \sum_{i \in S(I)} \exp \left(-\imath \mathbf{r} \cdot \boldsymbol{\Gamma}_{i}^{I}\right)$, while $\psi_{s}$ and $\psi_{l}$ are the equilibrium average densities of the crystal and the liquid, respectively. The chemical potential reads as

$$
\mu(\psi)=\left.\frac{\delta F}{\delta \psi}\right|_{\psi} .
$$

In this case, the equilibrium condition comes from the common tangent construction:

$$
\Delta \omega=\omega_{s}-\omega_{l}=0, \quad \text { and } \quad \mu_{s}=\mu_{l} .
$$

These two equations define the equilibrium solid and liquid densities, $\psi_{s}$ and $\psi_{l}$, respectively. Considering the $0^{\text {th }}$-order of $\Delta \omega=0$ and $\partial f_{s} / \partial A_{I}=0$ yields $q_{0}=1$, $y_{s}=y_{1}=1 / 2$ and $y_{I}=3 / 2$ for any $I>1$, where $y_{s}$ is the critical exponent of the solid equilibrium density, i.e. $\psi_{s}=c_{s} \cdot \epsilon^{y_{s}}$. Using these, $\mu_{s}=\mu_{l}$ starts with

$$
(1-\epsilon)\left(\psi_{s}-\psi_{l}\right)=0 \quad \Rightarrow \quad y_{l}=1 / 2, \quad c_{l}=c_{s},
$$

where $\psi_{l}=c_{l} \cdot \epsilon^{y_{l}}$ is the equilibrium liquid density. Note that $\psi_{l}$ and $\psi_{s}$ are equal in the leading order, i.e. $\psi_{s, l}=$ $c_{\psi} \cdot \epsilon^{y_{\psi}}$ again, where $y_{\psi}=1 / 2$. Therefore, $y_{\Delta}>1 / 2$ in $\Delta:=\psi_{s}-\psi_{l}=2 c_{\Delta} \cdot \epsilon^{y \Delta}$. Using $\psi_{l}=\bar{\psi}-\delta$ and $\psi_{s}=\bar{\psi}+\delta$ [where $\bar{\psi}:=\left(\psi_{l}+\psi_{s}\right) / 2=c_{\psi} \cdot \epsilon^{y_{\psi}}$ and $\left.\delta:=\left(\psi_{s}-\psi_{l}\right) / 2=c_{\Delta} \cdot \epsilon^{y \Delta}\right]$ in the next order of the equilibrium condition $\Delta \omega=0$ yields

$$
y_{\Delta}=3 / 2,
$$

which is the known mean-field result for crystal-liquid phase transitions.

\section{APPENDIX C: LEADING ORDER OF THE ANISOTROPIC INTERFACIAL FREE ENERGY}

\section{Neglecting the equilibrium density jump}

In order to evaluate the interfacial free energy, first we modify Eq. (17) as follows:

$$
\gamma(\mathbf{n}):=\int_{-\infty}^{\infty} d \xi\left(\frac{1}{A_{\perp}} \int_{\xi} d A_{\perp}\left\{\Delta I\left[\psi_{s l}\right]-\tau \cdot \Delta I\left[\psi_{p}\right]\right\}\right),
$$

where $\tau$ is to be determined later. Note that this modification is purely formal, since the contribution from $\Delta I\left[\psi_{p}\right]$ vanishes because of the equilibrium condition:

$$
\begin{aligned}
& \int_{-\infty}^{\infty} d \xi\left(\frac{1}{A_{\perp}} \int_{\xi} d A_{\perp}\left\{\Delta I\left[\psi_{p}(\mathbf{r})\right]\right\}\right) \propto \\
& \propto \int d V\left\{\Delta I\left[\psi_{p}\right]\right\} \propto \Delta f\left[\psi_{p}\right] \equiv 0 .
\end{aligned}
$$

For the sake of simplicity, first we introduce the shorthand notation

$$
\langle\cdot\rangle_{\xi}:=\left(1 / A_{\perp}\right) \int_{\xi} d A_{\perp}\{\cdot\} .
$$

Substituting Eq. (18) into Eq. (17), the terms appearing in the interfacial free energy can be expressed in the following general form:

$$
\int_{-\infty}^{\infty} d \xi\left\langle\prod_{I, i} \partial^{D_{i}^{I}} g_{i}^{I}(\xi) \prod_{J, j} h_{j}^{J}(\mathbf{r})\right\rangle_{\xi},
$$

where we used the shorthand notations $h_{j}^{J}(\mathbf{r})=\exp (\imath \mathbf{r}$. $\left.\boldsymbol{\Gamma}_{j}^{J}\right), \partial^{D_{i}^{I}} g_{i}^{I}(\xi)=\left[\partial^{D_{i}^{I}} / \partial\left(\xi / \Lambda_{i}^{I}\right)^{D_{i}^{I}}\right]\left[g\left(\xi / \Lambda_{i}^{I}\right)\right]$, and $\prod_{I, i}$ runs for some arbitrary RLVs. In order to evaluate Eq. (62) first we decompose the coordinate as $\mathbf{r}=\xi \cdot \mathbf{n}+\mathbf{r}_{\perp}$, where $\mathbf{r}_{\perp} \cdot \mathbf{n} \equiv 0$ (in other words, $\mathbf{r}_{\perp}$ is in the interface), resulting in the decoupled form of Eq. (62)

$$
\mathcal{I}_{\perp} \cdot \int_{-\infty}^{\infty} d \xi\left\{\prod_{I, i} \partial^{D_{i}^{I}} g_{i}^{I}(\xi) \prod_{J, j} h_{j}^{J}(\xi \cdot \mathbf{n})\right\},
$$

where

$$
\mathcal{I}_{\perp}=\left\langle\exp \left[\imath\left(\sum_{J, j} \boldsymbol{\Gamma}_{j}^{J}\right) \mathbf{r}_{\perp}\right]\right\rangle_{\xi}=\delta_{j_{1}, j_{2}, \ldots, j_{N}}^{J_{1}, J_{2}, \ldots, J_{N}}
$$

is not a function of $\xi$ anymore. Here we used the shorthand notation $\delta_{j_{1}, j_{2}, \ldots, j_{N}}^{J_{1}, J_{N}, \ldots, J_{N}}:=\delta\left(\sum_{n=1}^{N} \boldsymbol{\Gamma}_{j_{n}}^{J_{n}}\right)$. Note that if Eq. (64) gives 1, then $\prod_{J, j} h_{j}^{J}(\xi \cdot \mathbf{n})$ also gives 1, otherwise Eq. (63) is equal to 0 . Therefore, the term $\prod_{J, j} h_{j}^{J}(\xi \cdot \mathbf{n})$ in Eq. (63) can be omitted. Using the shorthand notation $\|\|:.=\int_{-\infty}^{\infty} d \xi\{$.$\} Eq. (62) can be re-written as:$

$$
\begin{aligned}
& \int_{-\infty}^{\infty} d \xi\left\langle\prod_{I, i} \partial^{D_{i}^{I}} g_{i}^{I}(\xi) \prod_{J, j} h_{j}^{J}(\mathbf{r})\right\rangle_{\xi}= \\
& =\left\|\prod_{I, i} \partial^{D_{i}^{I}} g_{i}^{I}(\xi)\right\| \cdot \delta_{j_{1}, j_{2}, \ldots, j_{N}}^{J_{1}, J_{2}, \ldots, j_{N}} .
\end{aligned}
$$

Note that this derivation is true only if $\mathbf{k}:=\sum_{j} \boldsymbol{\Gamma}_{j}^{J} \neq 0$ is not parallel with $\mathbf{n}$, otherwise $\mathbf{r}_{\perp} \cdot \mathbf{k} \equiv 0$. In this case $\int_{-\infty}^{+\infty} d x\left\{g\left(x / \Lambda_{i}^{I}\right) \cos \left(\Gamma_{j}^{J} x\right)\right\}$-type correction terms emerge, however, for $\Lambda_{i}^{I} \gg \Gamma_{j}^{J}$ (i.e. for $\epsilon \rightarrow 0$ ) they can be neglected.

Following the derivation presented in Appendix A, we can evaluate Eq. (70) as follows: First we introduce the shorthand notation $\langle.\rangle_{\gamma}:=\left\|\langle.\rangle_{\xi}\right\|$. Considering $\Delta I[\psi]=$ $I[\psi]-I[\bar{\psi}]$, where $I[$.$] is the integrand of Eq. (42) yields$

$$
\begin{aligned}
& \langle\Delta I[\psi]\rangle_{\gamma}=\frac{1}{2} \sum_{n=0}^{\infty} \alpha_{n}\left\langle\left(\nabla^{n} \Delta \psi\right)^{2}\right\rangle_{\gamma}+ \\
& +\frac{3 \bar{\psi}^{2}-\epsilon}{2}\left\langle\Delta \psi^{2}\right\rangle_{\gamma}+\bar{\psi}\left\langle\Delta \psi^{3}\right\rangle_{\gamma}+\frac{1}{4}\left\langle\Delta \psi^{4}\right\rangle_{\gamma},
\end{aligned}
$$

where $\psi$ can be either $\psi_{s l}$ or $\psi_{p}$. Introducing $\psi_{s l}=$ 
$\bar{\psi}+\Delta \psi_{s l}$ and $\psi_{p}=\bar{\psi}+\Delta \psi_{p}$ in Eq. (70) results in

$$
\begin{aligned}
\gamma(\mathbf{n}) & =\left\langle\Delta I\left[\psi_{s l}\right]\right\rangle_{\gamma}-\tau\left\langle\Delta I\left[\psi_{p}\right]\right\rangle_{\gamma}= \\
& =\frac{1}{2} \sum_{n=0}^{\infty} \alpha_{n}\left\langle\left(\nabla^{n} \Delta \psi_{s l}\right)^{2}\right\rangle_{\gamma}-\tau\left\langle\left(\nabla^{n} \Delta \psi_{p}\right)^{2}\right\rangle_{\gamma}+ \\
& +\frac{3 \bar{\psi}^{2}-\epsilon}{2}\left(\left\langle\Delta \psi_{s l}^{2}\right\rangle_{\gamma}-\tau\left\langle\Delta \psi_{p}^{2}\right\rangle_{\gamma}\right)+ \\
& +\bar{\psi}\left(\left\langle\Delta \psi_{s l}^{3}\right\rangle_{\gamma}-\tau\left\langle\Delta \psi_{p}^{3}\right\rangle_{\gamma}\right)+ \\
& +\frac{1}{4}\left(\left\langle\Delta \psi_{s l}^{4}\right\rangle_{\gamma}-\tau\left\langle\Delta \psi_{p}^{4}\right\rangle_{\gamma}\right)
\end{aligned}
$$

Using $\Delta \psi_{s l}=\sum_{I} A_{I} \sum_{i \in S(I)}\left[\left(1+g_{i}^{I}\right) / 2\right] h_{i}^{I}, \Delta \psi_{p}=$ $\sum_{I} A_{I} \sum_{i \in S(I)} h_{i}^{I}$ together with Eq. (65) in Eq. (67), and choosing $\tau=1 / 2$ yields

$$
\begin{aligned}
& \left\langle\Delta \psi_{s l}^{2}\right\rangle_{\gamma}-\frac{1}{2}\left\langle\Delta \psi_{p}^{2}\right\rangle_{\gamma}=\frac{1}{4} \sum_{I} A_{I}^{2} \sum_{i \in S(I)}\left\|\left(g_{i}^{I}\right)^{2}-1\right\| \\
& \left\langle\Delta \psi_{s l}^{3}\right\rangle_{\gamma}-\frac{1}{2}\left\langle\Delta \psi_{p}^{3}\right\rangle_{\gamma}= \\
& =\frac{1}{8} \sum_{I, J, K} A_{I} A_{J} A_{K} \sum_{i, j, k} \sum_{(m, n)}^{(i, j, k)}\left\|g_{m}^{M} g_{n}^{N}-1\right\| \delta_{i, j, k}^{I, J, K} \\
& \left\langle\Delta \psi_{s l}^{4}\right\rangle_{\gamma}-\frac{1}{2}\left\langle\Delta \psi_{p}^{4}\right\rangle_{\gamma}= \\
& =\frac{1}{16} \sum_{I, J, K} A_{I} A_{J} A_{K} A_{L}\left(\sum_{i, j, k, l}\left\|g_{i}^{I} g_{j}^{J} g_{k}^{K} g_{l}^{L}-1\right\|+\right. \\
& \left.+\sum_{(m, n)}^{(i, j, k, l)}\left\|g_{m}^{M} g_{n}^{N}-1\right\|\right) \delta_{i, j, k, l}^{I, J, K, L} .
\end{aligned}
$$

To find the first term of Eq. (67) we write

$$
\begin{aligned}
& \left\langle\left(\nabla \Delta \psi_{s l}\right)^{2}-\left(\nabla \Delta \psi_{p}\right)^{2}\right\rangle_{\gamma}= \\
& =\frac{1}{4} \sum_{I} A_{I}^{2} \sum_{i \in S(I)}\left[\left\|\left(g_{i}^{I}\right)^{2}-1\right\| \Gamma_{I}^{2}+\frac{\left\|\left(\partial g_{i}^{I}\right)^{2}\right\|}{\left(\Lambda_{i}^{I}\right)^{2}}\right] \\
& \left\langle\left(\nabla^{2} \Delta \psi_{s l}\right)^{2}-\left(\nabla^{2} \Delta \psi_{p}\right)^{2}\right\rangle_{\gamma}= \\
& =\frac{1}{4} \sum_{I} A_{I}^{2} \sum_{i \in S(I)}\left[\left\|\left(g_{i}^{I}\right)^{2}-1\right\| \Gamma_{I}^{4}-2 \frac{\left\|g_{i}^{I} \partial^{2} g_{i}^{I}\right\|}{\left(\Lambda_{i}^{I}\right)^{2}} \Gamma_{I}^{2}+\right. \\
& \left.+4\left(\mathbf{n} \cdot \Gamma_{i}^{I}\right)^{2} \frac{\left\|\left(\partial g_{i}^{I}\right)^{2}\right\|}{\left(\Lambda_{i}^{I}\right)^{2}}+O\left(1 / \Lambda^{3}\right)\right] \\
& \left\langle\left(\nabla^{3} \Delta \psi_{s l}\right)^{2}-\left(\nabla^{3} \Delta \psi_{p}\right)^{2}\right\rangle_{\gamma}= \\
& =\frac{1}{4} \sum_{I} A_{I}^{2} \sum_{i \in S(I)}\left[\left\|\left(g_{i}^{I}\right)^{2}-1\right\| \Gamma_{I}^{6}+\frac{\left\|\left(\partial g_{i}^{I}\right)^{2}\right\|}{\left(\Lambda_{i}^{I}\right)^{2}} \Gamma_{I}^{4}+\right. \\
& \left.+8\left(\mathbf{n} \cdot \Gamma_{i}^{I}\right)^{2} \Gamma_{I}^{2} \frac{\left\|\left(\partial g_{i}^{I}\right)^{2}\right\|}{\left(\Lambda_{i}^{I}\right)^{2}}+O\left(1 / \Lambda^{3}\right)\right] \\
& \left\langle\left(\nabla^{n} \Delta \psi_{s l}\right)^{2}-\left(\nabla^{n} \Delta \psi_{p}\right)^{2}\right\rangle_{\gamma}= \\
& =\frac{1}{4} \sum_{I} A_{I}^{2} \sum_{i \in S(I)}\left[\left\|\left(g_{i}^{I}\right)^{2}-1\right\| \Gamma_{I}^{2 n}+\right. \\
& \left.+\left(\propto \frac{\|\cdot\|}{\left(\Lambda_{i}^{I}\right)^{2}}\right)+O\left(1 / \Lambda^{3}\right)\right] .
\end{aligned}
$$

Substituting all the terms into Eq. (67) yields:

$$
\begin{aligned}
\gamma(\mathbf{n})= & \sum_{I} A_{I}^{2} \sum_{i \in S(I)}\left\{\frac{2 \zeta\left(\mathbf{n} \cdot \boldsymbol{\Gamma}_{i}^{I}, \Gamma_{I}\right)}{3 \Lambda_{i}^{I}}+\right. \\
& \left.+\frac{c_{2}\left(\Gamma_{I}\right)-\epsilon+3 \bar{\psi}^{2}}{8}\left\|\left(g_{i}^{I}\right)^{2}-1\right\|\right\}+ \\
& \frac{\bar{\psi}}{8} \sum_{I, J, K} A_{I} A_{J} A_{K} \sum_{i, j, k}\left[\sum_{(m, n)}^{(i, j, k)}\left\|g_{m}^{M} g_{n}^{N}-1\right\|\right] \delta_{i, j, k}^{I, J, K}+ \\
& \frac{1}{64} \sum_{I, J, K, L} A_{I} A_{J} A_{K} A_{L} \sum_{i, j, k, l}\left[\left\|g_{i}^{I} g_{j}^{J} g_{k}^{K} g_{l}^{L}-1\right\|+\right. \\
& \left.+\sum_{(m, n)}^{(i, j, k, l)}\left\|g_{m}^{M} g_{n}^{N}-1\right\|\right] \delta_{i, j, k, l}^{I, J, K, L},
\end{aligned}
$$

where we neglected the higher order terms $\left.\propto\left(\Lambda_{i}^{I}\right)^{-3}\right]$. Here $\zeta\left(\mathbf{n} \cdot \boldsymbol{\Gamma}_{i}^{I}, \Gamma_{I}\right)$ collects all terms proportional to $1 / \Lambda_{i}^{I}$ of Eq. (68). Note that the $c_{2}\left(\Gamma_{I}\right)$ term comes from the $\operatorname{sum} \sum_{n=0}^{\infty} \alpha_{n}\left\|\left(g_{i}^{I}\right)^{2}-1\right\| \Gamma_{I}^{2 n}=\left\|\left(g_{i}^{I}\right)^{2}-1\right\| c_{2}\left(\Gamma_{I}\right)$. Taking into account the result of Appendix D.1, i.e. that the critical exponents of the characteristic interface thicknesses must be equal, the leading order term of Eq. (69) is precisely Eq. (19).

\section{Including the equilibrium density jump}

Repeating the calculation for the case when the crystal-liquid equilibrium density jump is also considered 
is straightforward. In this case we use the definition of the surface tension:

$$
\begin{aligned}
\gamma^{\prime}(\mathbf{n}):= & \int_{-\infty}^{\infty} d \xi\left(\frac { 1 } { A _ { \perp } } \int _ { \xi } d A _ { \perp } \left\{\Delta I^{\prime}\left[\psi_{s l}\right]-\right.\right. \\
& \left.-\frac{1}{2} \cdot\left(\Delta I^{\prime}\left[\psi_{s}\right]+\Delta I^{\prime}\left[\psi_{l}\right]\right\}\right) .
\end{aligned}
$$

Here $\Delta I^{\prime}[\psi]=I^{\prime}[\psi]-I^{\prime}\left[\psi_{l}\right]$, where $I^{\prime}[\psi]=I[\psi]-$ $\mu \cdot \psi$ and $\mu=\delta F / \delta \psi$. Note that $\Delta I^{\prime}\left[\psi_{l}\right] \equiv 0$ and $\left(1 / A_{\perp}\right) \int d V \Delta I^{\prime}\left[\psi_{s}\right] \propto \Delta \omega\left[\psi_{s}\right]=0$ is the equilibrium condition. Furthermore, we use the following approximations:

$$
\begin{aligned}
\psi_{s l} & =\bar{\psi}+\Delta \varphi \sum_{i \in S(1)} g_{i}^{1}+\sum_{i \in S(1)} \frac{1+g_{i}^{1}}{2} h_{i}^{1} \\
\psi_{s} & =\bar{\psi}+\frac{\Delta}{2}+\sum_{i \in S(1)} h_{i}^{1}, \\
\psi_{l} & =\bar{\psi}-\frac{\Delta}{2}
\end{aligned}
$$

where $\Delta \varphi=\Delta / N_{1}$, i.e. the density jump $\Delta$ is distributed equally between the $N_{1}$ RLV vectors of the dominant RLV set. After a lengthy but straightforward calculation one can conclude to $\gamma^{\prime}(\mathbf{n})=\gamma(\mathbf{n})+O(\Delta)$, where $\gamma(\mathbf{n})$ is defined by Eq. (69) and $O(\Delta) \propto \epsilon^{5 / 2}$. Therefore, the equilibrium density jump has no contribution to the leading order of the interfacial free energy.

\section{APPENDIX D: THE ENVELOPE FUNCTION INTEGRALS}

\section{D.1: General properties}

In order to investigate the general, anisotropic case first we have to calculate $\left\|g_{i}^{I} g_{j}^{J}-1\right\|$ and $\left\|g_{i}^{I} g_{j}^{J} g_{k}^{K} g_{l}^{L}-1\right\|$ in Eq. (19). Unfortunately, no analytical formulae are known for these integrals as a function of the parameters $\Lambda_{i}^{1}, \Lambda_{j}^{1}, \Lambda_{k}^{1}$ and $\Lambda_{l}^{1}$. However, we can start from the integral $\left\|g_{i}^{I} g_{j}^{j}-1\right\|$ :

$$
\begin{aligned}
& \Lambda_{i}^{I} \int_{-\infty}^{\infty} d x\left[g(x) g\left(\frac{\Lambda_{i}^{I}}{\Lambda_{j}^{J}} x\right)-1\right] \equiv \\
& \equiv \Lambda_{j}^{J} \int_{-\infty}^{\infty} d y\left[g\left(\frac{\Lambda_{j}^{J}}{\Lambda_{i}^{I}} y\right) g(y)-1\right] .
\end{aligned}
$$

Introducing $f(\eta):=\int d x[\tanh (x) \tanh (\eta x)-1]$, where $\eta=\Lambda_{i}^{I} / \Lambda_{j}^{J}$ yields the following general constraint:

$$
\eta \cdot f[\eta]=f(1 / \eta), \quad \text { and } \quad f(1)=-2,
$$

which defines a family of functions for $f(\eta)$. More generally, we can use the following Ansatz:

$$
\left\|\prod_{k}^{n} g_{i_{k}}^{I_{k}}-1\right\| \approx \sum_{s}^{m} \frac{f_{s}^{(n)}}{\mathcal{N}\left[\mathbf{p}_{s}\right]} \sum_{l \in \mathcal{P}\left[\mathbf{p}_{s}\right]} \prod_{k}^{n}\left(\Lambda_{i_{k}}^{I_{k}}\right)^{p_{k(l)}^{(s)}}
$$

where $\sum_{l \in \mathcal{P}\left[\mathbf{p}_{s}\right]}$ runs over all permutations of the power set $\mathbf{p}_{s}=\left\{p_{1}^{(s)}, p_{2}^{(s)}, \ldots, p_{n}^{(s)}\right\}$ [i.e. $p_{k(l)}^{s}$ denotes the $k^{t h}$ element in the $l^{\text {th }}$ permutation of $\left.\mathbf{p}_{s}\right], \sum_{k=1}^{n} p_{k}^{(s)}=1$, $\mathcal{N}\left[\mathbf{p}_{s}\right]$ is the number of permutations, and the fitting parameters satisfy

$$
\sum_{s} f_{s}^{(n)}=\int_{-\infty}^{\infty} d x\left\{g^{n}(x)-1\right\}
$$

which can be calculated analytically. After choosing some power sets $\left\{\mathbf{p}_{s}\right\}$, the parameters $f_{s}^{(n)}$ can be determined via fitting the expression at such $\left(\Lambda_{i_{1}}^{I_{1}}, \Lambda_{i_{2}}^{I_{2}}, \ldots, \Lambda_{i_{N}}^{I_{N}}\right)$ points, for which the value of $\left\|\prod_{k}^{n} g_{i_{k}}^{I_{k}}-1\right\|$ is known. Considering that the critical behavior of Eq. (19) must be independent from the power sets used in Eq. (73) it is clear that all $x_{I}$ 's must be equal. [Otherwise, arbitrary powers of $\epsilon$ would emerge in Eq. (69)].

Considering only the leading order, the simplest form of $\left\|g_{i}^{1} g_{j}^{1}-1\right\|$ that couples $\lambda_{i}$ and $\lambda_{j}$ comes from the power sets $\mathbf{p}=(0,1)$ and $(1 / 2,1 / 2)$ :

$$
\left\|g_{i}^{1} g_{j}^{1}-1\right\| \approx \frac{f_{1}^{(2)}}{2}\left(\Lambda_{i}^{1}+\Lambda_{j}^{1}\right)+f_{2}^{(2)} \sqrt{\Lambda_{i}^{1} \Lambda_{j}^{1}} .
$$

$$
\text { D.2: Calculating the integrals for } g(.)=\tanh (.)
$$

For the particular case $g()=.\tanh (),. f_{1}^{(2)}+f_{2}^{(2)}=$ $\int_{-\infty}^{\infty} d x\left\{\tanh ^{2}(x)-1\right\}=-2$, Eq. (75) reduces to

$$
\left\|g_{i}^{1} g_{j}^{1}-1\right\| \approx H_{2}\left(\Lambda_{i}^{1}+\Lambda_{j}^{1}\right)-2\left(1+H_{2}\right) \sqrt{\Lambda_{i}^{1} \Lambda_{j}^{1}},
$$

where $\mathrm{H}_{2}$ can be determined by solving

$$
I_{2}[\eta]=H_{2}\left(1+\frac{1}{\eta}\right)-\frac{2\left(1+H_{2}\right)}{\sqrt{\eta}}
$$

for a chosen ratio $\eta=\Lambda_{i}^{1} / \Lambda_{j}^{1}=\lambda_{i}^{1} / \lambda_{j}^{1} \neq 1$, where $I_{2}[\eta]=$ $\int_{-\infty}^{+\infty} d x\{\tanh (x) \tanh (\eta \cdot x)-1\}$. The same derivation applies for $\left\|\left(g_{i}^{1} g_{j}^{1}\right)^{2}-1\right\|$, yielding

$$
\left\|\left(g_{i}^{1} g_{j}^{1}\right)^{2}-1\right\| \approx H_{4}\left(\Lambda_{i}^{1}+\Lambda_{j}^{1}\right)-2\left(\frac{4}{3}+H_{4}\right) \sqrt{\Lambda_{i}^{1} \Lambda_{j}^{1}},
$$

where $H_{4}$ can be determined via

$$
I_{4}[\eta]=H_{4}\left(1+\frac{1}{\eta}\right)-\frac{2\left(4 / 3+H_{4}\right)}{\sqrt{\eta}},
$$

where $I_{4}[\eta]=\int_{-\infty}^{+\infty} d x\left\{[\tanh (x) \tanh (\eta \cdot x)]^{2}-1\right\}$. Using Eq. (78) a reasonable approximation of $\left\|g_{i}^{1} g_{j}^{1} g_{k}^{1} g_{l}^{1}-1\right\|$ reads as:

$$
\begin{aligned}
\left\|g_{i}^{1} g_{j}^{1} g_{k}^{1} g_{l}^{1}-1\right\| \approx & \left(\frac{3 H_{4}}{4}+\frac{1}{3}\right)\left(\Lambda_{i}^{1}+\Lambda_{j}^{1}+\Lambda_{k}^{1}+\Lambda_{l}^{1}\right)- \\
& -\left(\frac{2}{3}+\frac{H_{4}}{2}\right) \sum_{m, n} \sqrt{\Lambda_{m}^{1} \Lambda_{n}^{1}} .
\end{aligned}
$$


Note that Eq. (80) reduces to $\left\|\left(g_{i}^{1} g_{j}^{1}\right)^{2}-1\right\|$ in case of two equal pairs in $\left\{\lambda_{i}, \lambda_{j}, \lambda_{k}, \lambda_{l}\right\}$.

Now we can evaluate the anisotropic interfacial free energy for the approximation $g()=.\tanh ($.$) as follows:$ First we calculate Eq. (19) divided by Eq. (34):

$$
\begin{aligned}
& \frac{\gamma(\mathbf{n})}{\gamma}=\sum_{i}\left[-\frac{3}{N_{1}} \tilde{\lambda}_{i}+\frac{\zeta\left(\mathbf{n} \cdot \boldsymbol{\Gamma}_{i}^{1}\right)}{2 C N_{1}} \frac{1}{\tilde{\lambda}_{i}}\right]+ \\
& \frac{3}{N_{3}} \sum_{i, j, k}\left[\sum_{m, n} f_{2}\left(\tilde{\lambda}_{m}, \tilde{\lambda}_{n}\right)\right] \delta_{i, j, k}^{1,1,1}- \\
& \frac{1}{N_{4}} \sum_{i, j, k, l}\left[f_{4}\left(\tilde{\lambda}_{i}, \tilde{\lambda}_{j}, \tilde{\lambda}_{k}, \tilde{\lambda}_{l}\right)+\frac{3}{4} \sum_{m, n} f_{2}\left(\tilde{\lambda}_{m}, \tilde{\lambda}_{n}\right)\right] \delta_{i, j, k, l}^{1,1,1,1},
\end{aligned}
$$

where $\tilde{\lambda}_{i}=\lambda_{i}^{1} / \lambda_{1}$ (the interface thickness relative to the isotropic solution $\Lambda_{1}=\lambda_{1} / \sqrt{\epsilon}$ ), whereas

$$
\begin{aligned}
f_{2}\left(\tilde{\lambda}_{i}, \tilde{\lambda}_{j}\right) & =\left\|g_{i}^{1} g_{j}^{1}-1\right\| /\left(-2 \Lambda_{1}\right) \\
f_{4}\left(\tilde{\lambda}_{i}, \tilde{\lambda}_{j}, \tilde{\lambda}_{k}, \tilde{\lambda}_{l}\right) & =\left\|g_{i}^{1} g_{j}^{1} g_{k}^{1} g_{l}^{1}-1\right\| /\left[(-8 / 3) \Lambda_{1}\right]
\end{aligned}
$$

\section{APPENDIX E: DETERMINING THE GINZBURG-LANDAU GRADIENT MATRICES}

First we modify Eq. (39) by subtracting (1/2) $\Delta f\left[\vec{\phi}_{S}\right]$ from the integrand in order to achieve finite surface contributions. Note that $\Delta f\left[\vec{\phi}_{S}\right] \equiv 0$, therefore this modification has no effect on Eq. (39).

Next, we assume that the planar equilibrium solution read as:

$$
\phi_{i}^{*}(\xi)=\frac{1+g_{i}(\xi)}{2}
$$

Using Eq. (84) and Eq. (37) in Eq. (39) results in

$$
\begin{aligned}
\gamma_{G L}(\mathbf{n})= & \frac{\kappa}{4} \sum_{i, j} s_{i j} \frac{\left\|\partial g_{i} \partial g_{j}\right\|}{\Lambda_{i} \Lambda_{j}}-\frac{w}{2 N_{1}} \sum_{i} \Lambda_{i}- \\
& -\frac{w}{4 N_{3}} \sum_{i, j, k}\left[\sum_{m, n}^{i, j, k}\left\|g_{m}^{M} g_{n}^{N}-1\right\|\right] \delta_{i, j, k}^{1,1,1}+ \\
& +\frac{w}{16 N_{4}} \sum_{i, j, k, l}\left[\left\|g_{i}^{I} g_{j}^{J} g_{k}^{K} g_{l}^{L}-1\right\|+\right. \\
& \left.+\sum_{m, n}\left\|g_{m}^{M} g_{n}^{N}-1\right\|\right] \delta_{i, j, k, l}^{1,1,1,1} .
\end{aligned}
$$

In addition, taking the leading order of Eq. (19), substituting $A_{1}=a_{1} \sqrt{\epsilon}, \bar{\psi}=c_{\psi} \sqrt{\epsilon}$ with Eqns. (15) and (16), and considering Eqns. (35) and (36) yields

$$
\begin{aligned}
\gamma_{P F C}(\mathbf{n})= & \sum_{i} \frac{\kappa}{C N_{1}} \frac{\zeta\left(\mathbf{n} \cdot \boldsymbol{\Gamma}_{i}^{1}\right)}{3 \Lambda_{i}}-\frac{w}{2 N_{1}} \sum_{i} \Lambda_{i}+ \\
& -\frac{w}{4 N_{3}} \sum_{i, j, k}\left[\sum_{m, n}^{i, j, k}\left\|g_{m}^{M} g_{n}^{N}-1\right\|\right] \delta_{i, j, k}^{1,1,1}+ \\
& +\frac{w}{16 N_{4}} \sum_{i, j, k, l}\left[\left\|g_{i}^{I} g_{j}^{J} g_{k}^{K} g_{l}^{L}-1\right\|+\right. \\
& \left.+\sum_{m, n}\left\|g_{m}^{M} g_{n}^{N}-1\right\|\right] \delta_{i, j, k, l}^{1,1,1,1} .
\end{aligned}
$$

Comparing Eq. (86) and (85) indicates that $\mathbb{S}$ must be diagonal, namely, $s_{i j}=s_{i} \delta_{i j}$, and $s_{i}=\zeta\left(\mathbf{n} \cdot \Gamma_{i}^{1}\right) /\left(N_{1} C\right)$. Note that $\sum_{i} s_{i i} \equiv 1$.

The corresponding coefficient matrices $\mathbb{A}_{i}$ in Eq. (40) can be determined as follows. First we express $\nabla \phi_{i}$ in an Euclidean coordinate system where the $x$ direction is parallel to the interface normal. Characterizing $\mathbf{n}$ by the $(\alpha, \beta, \gamma)$ Euler-angles yields the transformation matrix $\mathbb{R}=\mathbb{R}_{\alpha}^{z} \mathbb{R}_{\beta}^{y} \mathbb{R}_{\gamma}^{x}$, where $\mathbb{R}_{\delta}^{w}$ denotes a $3 \mathrm{D}$ rotation matrix by angle $\delta$ around axis $w$ in the original coordinate system. The gradient term can be then expressed as:

$$
\sum_{i} \nabla^{\prime} \phi_{i} \mathbb{M}_{i} \nabla^{\prime} \phi_{i}
$$

where $\mathbb{M}_{i}=\left(\mathbb{R}^{T} \cdot \mathbb{A}_{i} \cdot \mathbb{R}\right)$. For the planar equilibrium interface of normal $\mathbf{n}$ Eq. (87) reduces to

$$
\sum_{i} m_{11}^{(i)}\left(\partial_{\xi} \phi_{i}\right)^{2}
$$

where $m_{11}^{(i)}=\mathbf{n}^{T} \cdot \mathbb{A}_{i} \cdot \mathbf{n}$. Considering Eq. (39), $m_{11}^{(i)} \equiv$ $s_{i}=\left[\zeta_{0}+\zeta_{1} \cdot\left(\mathbf{n} \cdot \boldsymbol{\Gamma}_{i}^{1}\right)^{2}\right] /\left(N_{1} C\right)$ yields

$$
\mathbb{A}_{i}=\frac{1}{N_{1} C}\left[\zeta_{0} \cdot \mathbb{I}+\zeta_{1} \cdot\left(\boldsymbol{\Gamma}_{i}^{1} \otimes \boldsymbol{\Gamma}_{i}^{1}\right)\right] .
$$


[1] R. Napolitano, S. Liu, and R. Trivedi, Interface Science 10, 217 (2002).

[2] S. Liu, R. Napolitano, and R. Trivedi, Acta Materialia 49, 4271 (2001).

[3] R. E. Napolitano and S. Liu, Phys. Rev. B 70, 214103 (2004).

[4] J. Q. Broughton and G. H. Gilmer, The Journal of Chemical Physics 84, 5759 (1986).

[5] R. L. Davidchack and B. B. Laird, Phys. Rev. Lett. 85, 4751 (2000).

[6] V. G. Baidakov, S. P. Protsenko, and A. O. Tipeev, The Journal of Chemical Physics 139, 224703 (2013).

[7] R. L. Davidchack and B. B. Laird, Phys. Rev. Lett. 94, $086102(2005)$.

[8] J. J. Hoyt, M. Asta, and A. Karma, Phys. Rev. Lett. 86, 5530 (2001).

[9] J. R. Morris, Phys. Rev. B 66, 144104 (2002).

[10] D. Y. Sun, M. Asta, J. J. Hoyt, M. I. Mendelev, and D. J. Srolovitz, Phys. Rev. B 69, 020102 (2004).

[11] D. Y. Sun, M. Asta, and J. J. Hoyt, Phys. Rev. B 69, 174103 (2004).

[12] A. D. J. Haymet and D. W. Oxtoby, J. Chem. Phys. 74, 2559 (1981).

[13] D. W. Oxtoby and A. D. J. Haymet, J. Chem. Phys. 76, 6262 (1982).

[14] T. V. Ramakrishnan and M. Yussouff, Phys. Rev. B 19, 2775 (1979).

[15] W. H. Shih, Z. Q. Wang, X. C. Zeng, and D. Stroud, Phys. Rev. A 35, 2611 (1987).
[16] S. A. Brazovskii, I. E. Dzialoshinskii, and A. R. Muratov, Zh. Eksp. Teor. Fiz. 93, 1110 (1987).

[17] K.-A. Wu, A. Karma, J. J. Hoyt, and M. Asta, Phys. Rev. B 73, 094101 (2006).

[18] K. R. Elder, M. Katakowski, M. Haataja, and M. Grant, Phys. Rev. Lett. 88, 245701 (2002).

[19] K.-A. Wu and A. Karma, Phys. Rev. B 76, 184107 (2007).

[20] A. Jaatinen, C. V. Achim, K. R. Elder, and T. AlaNissila, Phys. Rev. E 80, 031602 (2009).

[21] S. Majaniemi and N. Provatas, Phys. Rev. E 79, 011607 (2009).

[22] K. R. Elder, N. Provatas, J. Berry, P. Stefanovic, and M. Grant, Phys. Rev. B 75, 064107 (2007).

[23] K. R. Elder and M. Grant, Phys. Rev. E 70, 051605 (2004).

[24] F. Podmaniczky, G. I. Tóth, T. Pusztai, and L. Gránásy, Journal of Crystal Growth 385, 148 (2014), the 7th International Workshop on Modeling in Crystal Growth.

[25] H. Emmerich, L. Gránásy, and H. Löwen, The European Physical Journal Plus 126, 102 (2011), 10.1140/epjp/i2011-11102-1.

[26] J. Kundin, M. Choudhary, and H. Emmerich, The European Physical Journal Special Topics 223, 363 (2014).

[27] M. Greenwood, N. Provatas, and J. Rottler, Phys. Rev. Lett. 105, 045702 (2010).

[28] G. I. Tóth, L. Gránásy, and G. Tegze, Journal of Physics: Condensed Matter 26, 055001 (2014). 